\title{
Culture of Dendritic Cells from a Nonlymphoid Organ, the Thyroid Gland: Evidence for TNF $\alpha$-Dependent Phenotypic Changes of Thyroid-Derived Dendritic Cells
}

\author{
Karine Croizet, Rachida Rabilloud, Zdenek Kostrouch, Jean-Francois Nicolas, and \\ Bernard Rousset \\ INSERM, U-369 (KC, RR, ZK, BR), and INSERM, U-503 (J-FN), Faculté de Médecine, Lyon-RTH Laennec, France
}

\begin{abstract}
SUMMARY: Because they are sparsely distributed in tissues, dendritic cells (DC) present in nonlymphoid organs are difficult to isolate. Only DC from skin and lung have been successfully studied in culture. The objective of the present work was to investigate the possibility of isolating and culturing DC from an endocrine organ, the thyroid gland, which is particularly susceptible to the development of autoimmune processes. The study was conducted on pig thyroid glands to have sufficient amounts of starting material. This choice required the characterization of immunological reagents capable of recognizing DC markers in the pig species. Using a discontinuous trypsinization procedure, a DC population representing $2 \%$ to $3 \%$ of the thyroid cell suspension was reproducibly obtained. Isolated DC quantitatively attached to tissue culture-treated dishes and segregated from thyrocytes. DC identified as cells expressing major histocompatibility complex class II molecules, the mannose receptor, and the S100 protein were found to have a high capacity to internalize labeled ligands, dextran, and mannosylated albumin. These cells had a phenotype of immature DC. Secondarily, a fraction of DC detached from culture dishes, and floating DC had low or no endocytic activity, a characteristic of mature DC. Treatment of DC/thyrocytes cocultures with tumor necrosis factor $\alpha$ (TNF $\alpha$ ) activated the transformation of immature DC into mature DC. These data show that DC isolated from the thyroid gland can be maintained immature or activated to undergo maturation in primary culture. The procedure of cell isolation and culture should be adaptable to human thyroid tissue for in vitro analyses of DC-mediated immune responses. (Lab Invest 2000, 80:1215-1225).
\end{abstract}

$D$ endritic cells (DC) are the most potent antigenpresenting cells of the immune system and are critically involved in the initiation of primary immune responses, the generation of T-cell-dependent antibodies, graft rejection, and autoimmune diseases (Steinman, 1991; Winzler et al, 1997). DC are scattered throughout the body in many nonlymphoid organs where they exert a sentinel function. DC present in nonlymphoid organs, also named immature interstitial DC, are capable of capturing and processing antigens. In response to a stimulus, interstitial DC undergo maturation; they lose their antigen-capturing capacity, acquire T-cell stimulatory capabilities and migrate to the T-dependent areas of lymphoid organs where they efficiently present antigen to T-cells.

Cells exhibiting morphological and immunological features of interstitial DC have been identified in the normal mouse thyroid gland (Hart and Fabre, 1981; Hart et al, 1983; Kabel et al, 1988). It has been reported that intrathyroidal DC could be involved in the early stages of autoimmune thyroid diseases in humans (Kabel et al, 1988; Mölne et al, 1994) and in experimental autoimmune thyroiditis in mouse (Knight et al, 1988; Many et al, 1995) and rat models (Simons

Received February 23, 2000.

Address reprint requests to: Prof. Bernard Rousset, INSERM U369, Faculté de Médecine RTH Laennec, 7, Rue Guillaume Paradin, 69372 LYON Cedex 08, France.Fax: 3347877 8736; E-mail: u369@laennec.univlyon1.fr et al, 1998; Voorby et al, 1990). As DC are sparsely distributed in the gland and thus difficult to isolate, these studies were restricted to analyses of DC on tissue sections. Among DC originating from nonlymphoid tissues, only epidermal DC, or Langerhans cells, and lung DC have been isolated and cultured as enriched DC populations (Masten and Lipscomb, 1999; Schuler and Steinman, 1985). Here, we report that a discontinuous trypsinization procedure used to isolate thyroid epithelial cells or thyrocytes led to the simultaneous isolation of a population of DC that was successfully maintained in primary culture with thyrocytes. DC extracted from the thyroid gland, identified as cells expressing major histocompatibility complex (MHC) class II determinants (Banchereau and Steinman, 1998; Steinman, 1991), the mannose receptor (Sallusto et al, 1995), and the S100 protein (Caux et al, 1996), exhibited a high capacity to internalize various ligands. By analyzing their endocytic activity and adherence properties, it was found that DC, in a immature state at the initiation of the primary culture, subsequently undergo maturation.

\section{Results}

Identification of DC in Pig Thyroid Tissue and in the Cell Suspension Obtained by Proteolytic Treatment

To facilitate the isolation of DC that represent a small cell population within the thyroid gland, the study was conducted on pig thyroid glands that were available in 
larger amounts than either human, mouse or rat thyroid tissues. It was thus necessary to find immunological reagents capable of identifying DC markers in the porcine species. After a laborious search, we selected a monoclonal antibody directed against the bovine $\mathrm{S} 100 \beta$ protein reacting with the human and porcine $\mathrm{S} 100 \beta$ and two monoclonal antibodies directed against human antigens: MHC class II human leukocyte antigen (HLA)-DR molecules and the mannose receptor that were found to react specifically with the corresponding molecules from pig tissues. The antihuman HLA class II antibody identified a $28 \mathrm{kDa}$ component in pig (results not shown). Using human lung alveolar macrophages as reference material, a $175 \mathrm{kDa}$ protein corresponding to the mannose receptor (Drickamer and Taylor, 1993; Stahl, 1992) was detected in human, rat, and pig thyroid crude membrane extracts by the antihuman mannose receptor monoclonal antibody (Fig. 1). These antibodies were used to identify cells expressing $\mathrm{MHC}$ class II molecules and the mannose receptor in pig thyroid cryosections by indirect immunofluorescence. Results are reported in Figure $2, A_{a}$ to $A_{d}$. Cells positive for the mannose receptor $\left(A_{a}\right)$ or $\mathrm{MHC}$ class II $\left(A_{c}\right)$ were found in the interstitial or conjunctive tissue between layers of epithelial thyroid cells (identified by Hoechst labeling of nuclei) delimiting follicle structures. Although difficult to determine with accuracy because of the thickness of the cryosections, the proportion of $\mathrm{MHC}$ class II or mannose receptor-positive cells within the intact pig thyroid tissue was estimated at between $2 \%$ and $4 \%$.

To detect the presence of cells exhibiting DC markers within the cell population resulting from the proteolytic treatment, cells were spread on glass slides by cytocentrifugation and subjected to a double labeling by the Hoechst fluorescent reagent to identify all the nuclei and by indirect immunofluorescence with either the anti-MHC class II or the antimannose receptor antibodies. MHC class II-positive cells represented $2.2 \pm 0.3 \%(n=3)$ of the total cell population. Most of

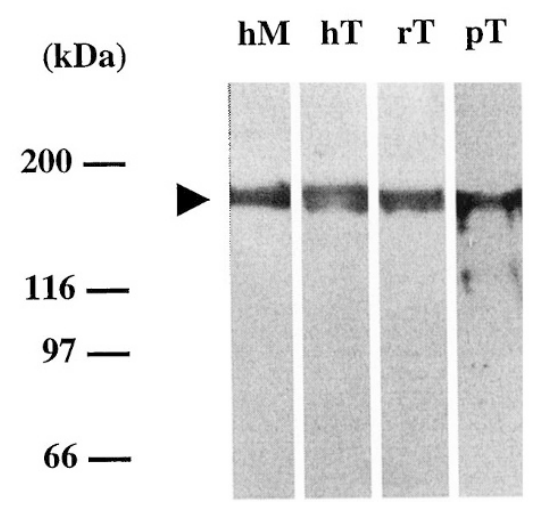

these cells were recognizable after Hoechst labeling. They exhibit a reniform nucleus (Fig. 2, panels B). Unexpectedly, the cell population isolated from the thyroid tissue did not contain mannose receptorpositive cells. Attempts to identify the mannose receptor on isolated cell membrane extracts by Western blot were also unsuccessful (Fig. 1). In some experiments, the antimannose receptor monoclonal antibody labeled a minor 90 to $100 \mathrm{kDa}$ component, likely representing a proteolytic fragment. Because the extracellular domain of the receptor has been reported to be sensitive to proteolytic cleavage reactions (Martinez-Pomares et al, 1998), the lack of detection of the mannose receptor on DC present in the freshly dispersed cell suspensions probably resulted from the action of proteolytic enzymes used to dissociate cells from the tissue.

\section{Characterization and Functional Properties of Thyroid-Derived DC in Coculture with Thyrocytes}

After 3 to 4 days of culture, thyrocytes did not form a cell monolayer but organized into three-dimensional spherical structures or reconstituted thyroid follicles (Munari-Silem et al, 1990). Indirect immunofluorescence experiments with either anti-MHC class II or antimannose receptor antibodies revealed the presence of labeled cells scattered in between follicle structures over the entire surface of Petri dishes. As shown in Figure 3, A and B, mannose receptorpositive cells or MHC class II-positive cells were generally larger than thyrocytes and were either round or elongated, but always displayed cytoplasmic extensions. These cells did not participate in the constitution of follicles. However, in some cases, MHC class II or mannose receptor-positive cells were found close to thyroid cell aggregates indicating that contacts with thyrocytes might occur. To exclude the possibility that these cells could represent a subpopulation of thyrocytes with a peculiar morphological phenotype, we determined whether they expressed the thyroid cell

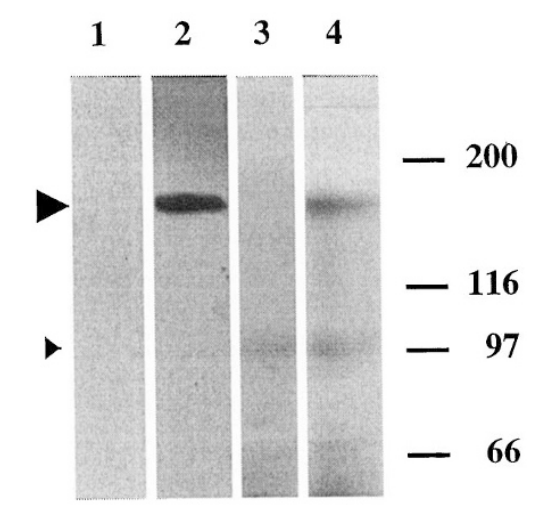

\section{Figure 1.}

Identification of the mannose receptor by western blot. Membrane proteins from crude particulate fractions, obtained by centrifugation of cell lysates or tissue homogenates at $100,000 \times g$ for 30 minutes, were fractionated by SDS-PAGE (6\% acrylamide gels) and transferred on Immobilon P membranes for western blotting using the antimannose receptor monoclonal antibody (final concentration, $0.1 \mu \mathrm{g} / \mathrm{ml}$ ). Samples were membrane protein $(3 \mu \mathrm{g})$ from human lung macrophages (hM) and membrane protein $(50 \mu \mathrm{g})$ from human $(\mathrm{hT})$, rat $(\mathrm{rT})$, and pig $(\mathrm{pT})$ thyroid tissue, respectively. Lanes 1 and $2,80 \mu \mathrm{g}$ membrane protein from freshly dispersed thyroid cells and corresponding cells after 4 days of culture; lanes 3 and 4 , as above but with a different cell preparation. Arrows identify the 175 kDa mannose receptor and a potential cleavage product. The position and size (in $\mathrm{kDa}$ ) of the molecular mass standards are indicated on each side of the figure. 

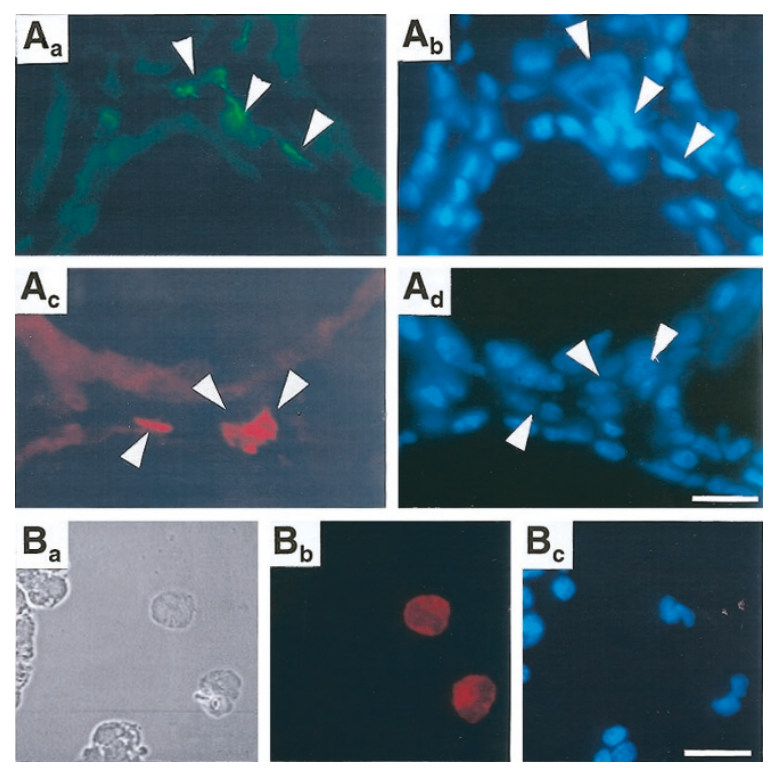

\section{Figure 2.}

Identification of mannose receptor and major histocompatibility complex (MHC) class II-positive cells in thyroid tissue cryosections (A) and in freshly dispersed thyroid cell suspensions (B). A, Cryosections prepared from pig thyroid tissue were subjected to indirect immunofluorescence labeling with either the antimannose receptor antibody $\left(A_{a}\right)$ or the anti-MHC class II antibody $\left(A_{c}\right) . A_{b}$ and $A_{d}$ give the distribution of cells (visualized by Hoechst labeling of nuclei) in cryosections presented in $\mathrm{A}_{a}$ and $\mathrm{A}_{c}$, respectively. Arrows indicate the position of cells expressing the mannose receptor or MHC class II molecules. B, Freshly dispersed thyroid cells spread on glass slides by cytocentrifugation were immunolabeled with the anti-MHC class II antibody. $B_{a}$, Phase contrast image; $B_{b}$, MHC class II labeling; $B_{c}$, Hoechst labeling of nuclei. Noteworthy, MHC class II-positive cells exhibit a polymorphous nucleus. Bars $=15 \mu \mathrm{m}$.

marker, thyroglobulin. Antithyroglobulin antibodies strongly labeled cells involved in the constitution of follicle structures, but cells exhibiting DC features were always thyroglobulin negative (result not shown).

As one of the main functions of DC is to ingest and process antigens, we examined the endocytic activity of thyroid-derived cells after 2 to 4 days in primary culture. After a 3-hour incubation at $37^{\circ} \mathrm{C}$ in the presence of fluorescein-labeled dextran and rhodamine-labeled mannosylated bovine serum albumin (Rh-ManBSA), cells participating in the constitution of follicle structures were slightly fluorescent, an observation in keeping with previous results (Gire et al, 1996) on fluid phase basolateral endocytosis of thyrocytes. In contrast, cells distributed in between follicles exhibited a strong fluorescence. This is illustrated in Figure $3, C_{a}$ to $C_{d}$. Superimposition of fluoresceinlabeled dextran (F-Dx) and Rh-ManBSA labelings (Fig. $3 \mathrm{C}_{\mathrm{c}}$ ) indicated that the two fluorescent probes were internalized by the same cells and that F-Dx and Rh-ManBSA were present in the same vacuolar structures, probably corresponding to endocytic compartments. Cells with a round shape exhibited membrane ruffles with spikes. Elongated cells presented large lamellipodiae and sometimes very long cytoplasmic projections resembling dendrites. When the incubation was performed at $4^{\circ} \mathrm{C}$, cells were no longer labeled, indicating that fluorescence labeling was re-
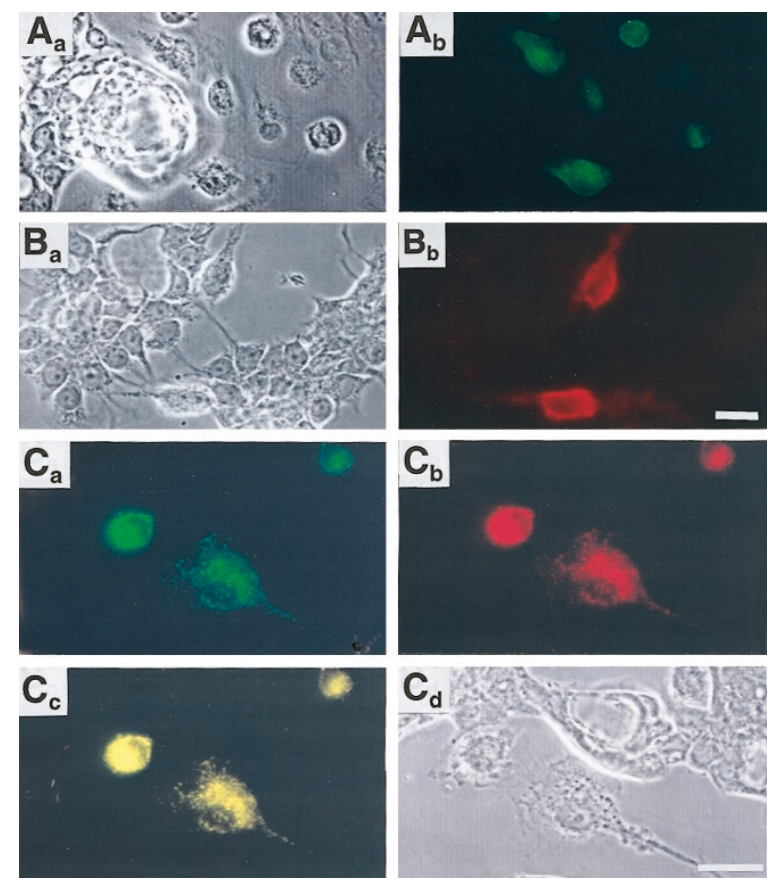

\section{Figure 3.}

Identification of mannose receptor- and MHC class II-positive cells in primary cultures of thyroid cells and detection of cells with a high endocytic activity. Cells cultured for 4 days were either subjected to indirect immunofluorescence staining with the antimannose receptor antibody (A) or the anti-MHC class II antibody (B) or incubated with fluorescent ligands, fluorescein-labeled dextran (F-Dx) and rhodamine-labeled mannosylated BSA (Rh-Man BSA), each at a final concentration of $1 \mathrm{mg} / \mathrm{ml}$ for 3 hours at $37^{\circ} \mathrm{C}(\mathrm{C})$. Phase contrast and fluorescence images were taken sequentially. $A_{a}$ and $A_{b}$, Phase contrast and fluorescence images of a microscope field after labeling with the antimannose receptor antibody. $B_{a}$ and $B_{b}$, Phase contrast and fluorescence images of a field after labeling with the anti-MHC class II antibody. $C_{a}$ and $C_{b}$, Fluorescence images of cells that internalized both F-Dx $\left(C_{a}\right)$ and Rh-Man BSA $\left(C_{b}\right) ; C_{c}$, superimposition of $C_{a}$ and $C_{b}$ fluorescence images; $C_{d}$, phase contrast image of the corresponding field. Labeled cells were located in between groups of cells corresponding to thyrocytes organized in follicle structures. Noteworthy, because of cell fixation, reconstituted follicules have lost most of their three-dimensional morphological characteristics and appear more as cell aggregates; however, in most cases a space corresponding to the follicle lumen can be identified. Bar $=15 \mu \mathrm{m}$.

lated to the internalization of F-Dx and Rh-Man BSA. Cells with the high endocytic activity, ie, F-Dx- and/or Rh-ManBSA-labeled cells, amounted to $2.0 \pm 0.3 \%$ $(n=7)$ of total cells (numbered after Hoechst reagent labeling of nuclei). The morphological characteristics of cells with a high endocytic activity were further analyzed by transmission electron microscopy after an incubation in the presence of protein-gold particles. Some BSA-gold and thyroglobulin-gold particles were detected in intracellular compartments of thyrocytes having the characteristics of late endosomes and lysosomes as previously reported (Gire et al, 1996), but they were found in much higher amounts in cells corresponding (in terms of shape and localization in petri dishes) to cells internalizing fluorescent ligands at a high rate. These cells contained several hundreds of gold-particles located in electron-dense compartments. Figure 4A shows such a cell neighboring a follicle identifiable by its luminal compartment delimited by a single layer of polarized cells. At higher 

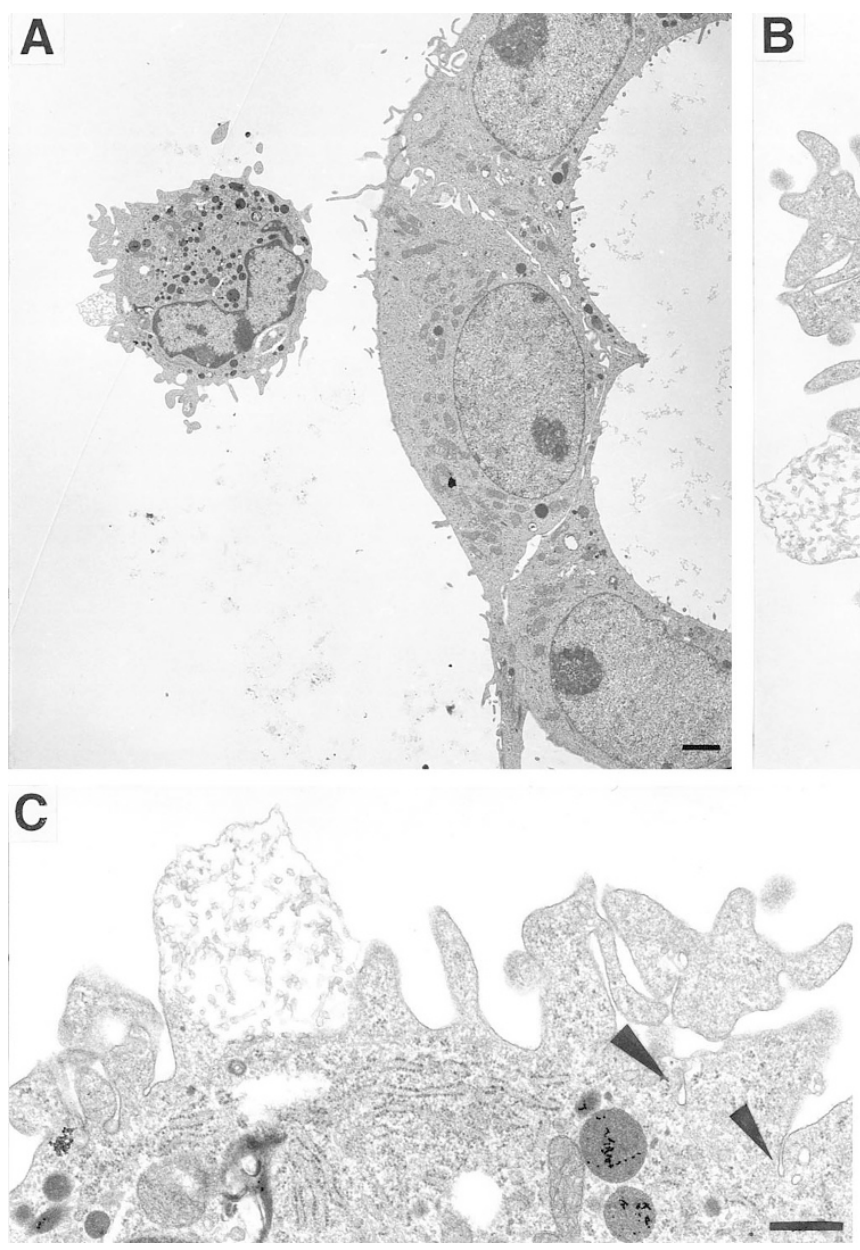
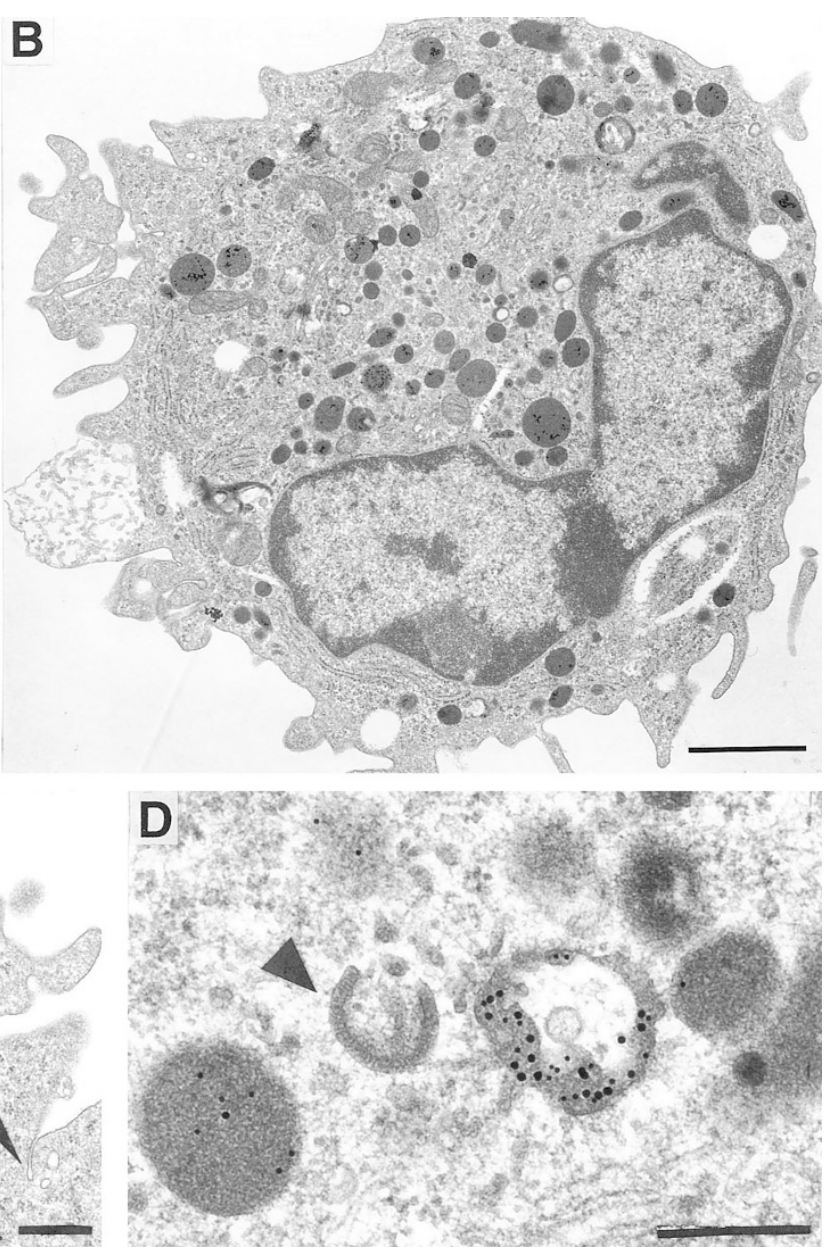

\section{Figure 4.}

Transmission electron microscope examinations of cells endowed with a high endocytic activity. Cells cultured for 4 days were incubated with BSA-gold and thyroglobulin-gold complexes and processed as indicated in the section "Materials and Methods." A, Cell with a high capacity to internalize gold particles in the vicinity of a follicule structure. B, Higher magnification of the same cell exhibiting a reniform nucleus, membrane ruffles, and electron dense vacuoles containing a large number of gold particles. C, Enlargement of a portion of the cell showing structures resembling Birbeck granules (arrowheads). D, Portion of another cell with vacuolar compartments (probably prelysosomes and lysosomes) containing BSA-gold and thyroglobulin-gold particles; the arrowhead indicates an annular structure commonly observed in antigen-presenting cells. Bars $=1.5 \mu \mathrm{m}$ in $A$ and $B$ and $500 \mathrm{~nm}$ in $C$ and D.

magnifications (Fig. 4, C and D), the two types of protein gold particles were observed in vacuolar structures corresponding to endosomes and lysosomes. Compared with thyrocytes, cells full of gold particles (Fig. 4B), (a) contained many more electron-dense structures, (b) had a plasma membrane with numerous processes, and (c) exhibited a reniform nucleus. By examining ultrathin sections of numerous cells endowed with high endocytic capacity, we noticed the presence of plasma membrane domains where the lipid bilayer joined side by side (Fig. 4C). These peculiar structures could be reminiscent of Birbeck granules. Furthermore, we observed intracellular annular or horseshoe-shaped structures (Fig. 4D) that have been described in antigen-presenting cells.

To investigate whether cells with a high endocytic activity corresponded to MHC class II and mannose receptor-positive cells, a two-step labeling procedure was used. Cells were first incubated with either F-Dx or Rh-ManBSA and then submitted to the indirect immunofluorescence staining procedure. Fluores- cence images presented in Figure 5A show that cells internalizing Rh-ManBSA (panel $A_{a}$ ) were strongly mannose receptor-positive (panel $A_{b}$ ). Intracellular compartments accumulating labeled Man-BSA were, for most of them, mannose receptor-positive. This is in keeping with the presence of mannose receptor molecules at the plasma membrane and in endocytic and recycling compartments (Lennartz et al, 1989; Stahl et al, 1980). Figure 5B shows that F-Dx-positive cells (panel $B_{a}$ ) were MHC class II-positive (panel $B_{b}$ ). Image superimposition (panel $\mathrm{B}_{\mathrm{c}}$ ) showed no colocalization; MHC class II labeling appeared diffuse over the whole cell surface. MHC class II molecules were indeed present at the plasma membrane because fluorescence distribution was very similar with or without cell permeabilization (data not shown). Noteworthy, MHC class II molecules were expressed only by cells internalizing high amounts of F-Dx; thyroid cells, visualized by Hoechst labeling of nuclei, were $\mathrm{MHC}$ class II-negative (panel $\mathrm{B}_{\mathrm{d}}$ ). To insure that $\mathrm{MHC}$ class II and mannose receptor-positive cells endowed with 

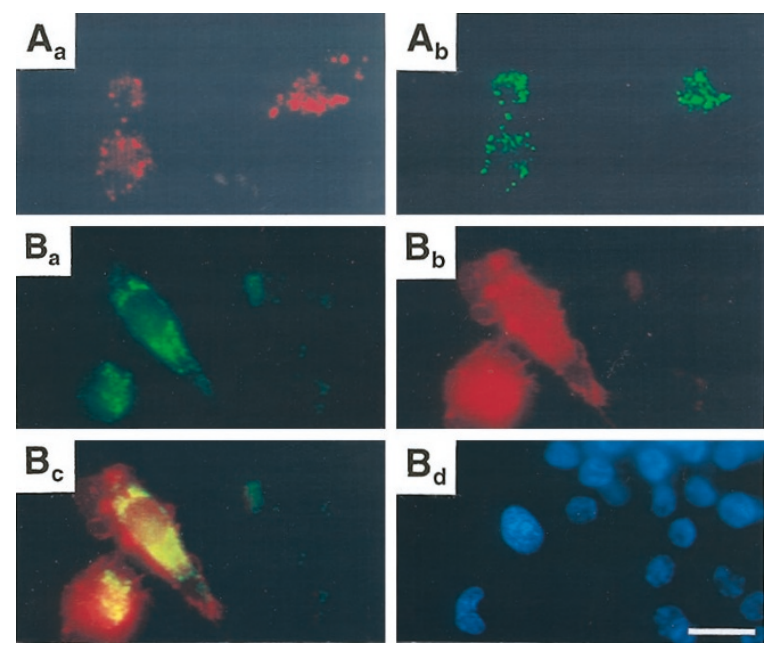

Figure 5.

Evidence that cells internalizing F-Dx and/or Rh-ManBSA are mannose receptor- and MHC class II-positive cells. Cells cultured for 4 days were incubated with F-Dx or Rh-ManBSA for 3 hours at $37^{\circ} \mathrm{C}$ and then fixed and subjected to indirect immunofluorescence labeling using monoclonal antibodies directed against either the mannose receptor or MHC class II molecules. Immune complexes were detected with goat antimouse IgG conjugated to FITC or Texas red. A, Cells with the capacity to internalize Rh-ManBSA $\left(A_{a}\right)$ were mannose receptor-positive $\left(A_{b}\right)$. B, Cells that internalize $F-D x\left(B_{a}\right)$ were labeled by the anti-MHC class II antibody $\left(B_{b}\right)$. The superimposition of $B_{a}$ and $B_{b}$ images in $B_{c}$ shows the location of endocytic compartments within MHC class II-positive cells. The labeling of nuclei by the Hoechst reagent $\left(B_{d}\right)$ allows us to see that the others cells (ie, thyrocytes) were MHC class II-negative. Bar = $15 \mu \mathrm{m}$.

a high endocytic activity actually correspond to DC, we used another marker, the $\mathrm{S} 100 \beta$ protein (Caux et al, 1996). This required a special double immunofluorescence labeling procedure (Negoescu et al, 1994) adapted to the sequential use of two mouse monoclonal antibodies (anti-MHC class II and anti-S100 $\beta$ monoclonal antibodies). Representative images are presented in Figure 6. MHC class II-positive cells (panel E) were also clearly $\mathrm{S} 100 \beta$-positive (panel F).

To determine whether all cells with a high endocytic activity corresponded to DC, cells cultured for 4 days were subjected to a series of double fluorescence labelings designated in Table 1 as Marker 1 and Marker 2. The four labelings were (a) co-incubation with F-Dx and Rh-Man BSA, (b) incubation with F-Dx and immunodetection of MHC class II, (c) incubation with Rh-Man BSA and immunodetection of the mannose receptor, and (d) sequential immunodetection of $\mathrm{MHC}$ class II and S100 $\beta$ protein. Images corresponding to each fluorphore (fluorescein and either rhodamine or Texas red) were recorded sequentially. Cells labeled with only one fluorphore and double-labeled cells were counted on 10 microscope fields (containing between 30 and 50 labeled cells). Cell counts were used to determine the percentage of Marker 2-positive cells within the Marker 1-positive cell population. Values obtained from duplicate or triplicate dishes in two to four separate cultures were pooled and used to calculate the mean \pm SEM ( $n$, number of measurements). Data summarized in Table 1 show that, except in one case, more than $90 \%$ of cells internalizing F-Dx
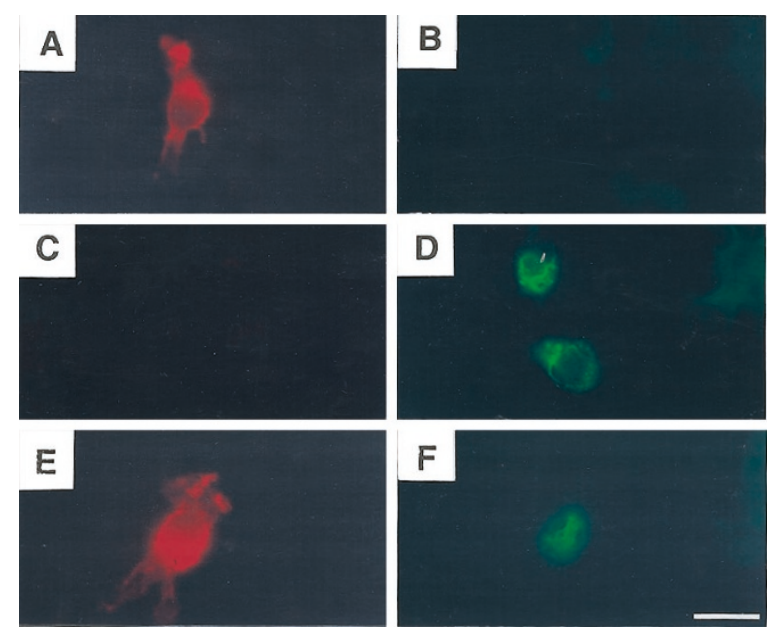

\section{Figure 6.}

Double immunofluorescence labeling showing that MHC class II-positive cells were $S 100 \beta$-positive. Cells cultured for 4 days were subjected to a labeling procedure (see "Materials and Methods" section) developed for the sequential use of two mouse monoclonal antibodies (anti-MHC class II and anti-S100 $\beta$ ), the detection of immune complexes being sequentially performed using the same secondary antibody conjugated to Texas red (first labeling) or to FITC (second labeling). A to $\mathrm{D}$ represent controls of the double immunolabeling shown in $E$ (MHC class II) and F (S100 $\beta$ protein). A and B, Double labeling with omission of the second primary antibody (anti-S100B); $C$ and D, double labeling with omission of the first primary antibody (anti-MHC class II). Bar = $15 \mu \mathrm{m}$.

or Rh-ManBSA expressed the mannose receptor, $\mathrm{MHC}$ class II molecules, and the $\mathrm{S} 100 \beta$ protein. Values ranging from $90 \%$ to $97 \%$ were not statistically different from $100 \%$. However, we reproducibly found a population of cells representing up to $10 \%$ to $15 \%$ of cells with the high endocytic activity that did not express detectable levels of MHC class II molecules.

\section{Phenotypic Changes of DC in Primary Culture: Effects of TNF $\alpha$}

Examinations of culture media after different periods of culture revealed the presence of $\mathrm{MHC}$ class IIpositive cells within the floating cell population. MHC
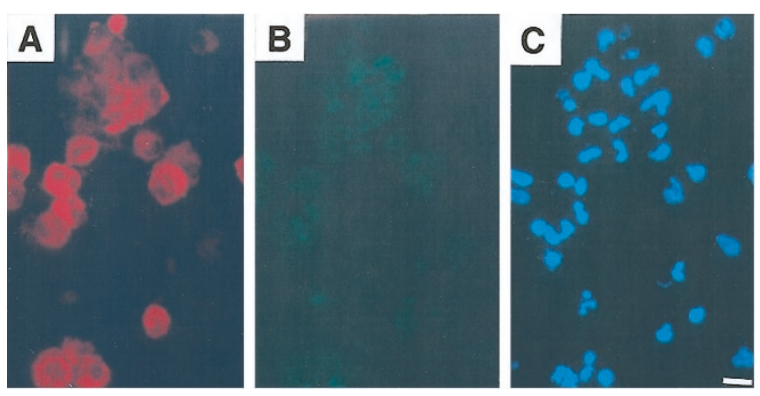

Figure 7.

Identification of MHC class II-positive cells in the medium of thyrocytes/DC cocultures. Cells cultured for 4 days were incubated with F-Dx for 3 hours at $37^{\circ} \mathrm{C}$. At the end of the incubation period, cells present in the culture medium were collected, spread on glass slides by cytocentrifugation, and immunolabeled with the anti-MHC class II antibody. A to C, Fluorescence images of MHC class II labeling, F-Dx internalization, and Hoechst-labeled nuclei of the same microscope field. Bar $=15 \mu \mathrm{m}$. 
Table 1. Proportion of Marker 2-Positive Cells within the Marker 1-Positive Cell Population (\%)

\begin{tabular}{lccccc}
\hline & \multicolumn{5}{c}{ Marker 2} \\
\cline { 2 - 5 } Marker 1 & F-Dx & Rh-ManBSA & Mannose receptor & MHC II & S100 \\
\hline F-Dx & - & $94 \pm 4_{(\mathrm{n}=6)}$ & - & $82 \pm 6_{(\mathrm{n}=6)}$ & - \\
Rh-ManBSA & $94 \pm 3_{(\mathrm{n}=6)}$ & - & $90 \pm 8_{(\mathrm{n}=4)}$ & - & - \\
Mannose receptor & - & $93 \pm 5_{(\mathrm{n}=4)}$ & - & - & - \\
MHC II & $95 \pm 7_{(\mathrm{n}=6)}$ & - & - & - & $97 \pm 8_{(\mathrm{n}=9)}$ \\
S100 $\beta$ & - & - & - & $92 \pm 7_{(\mathrm{n}=9)}$ & - \\
\hline
\end{tabular}

F-Dx, fluorescein-labeled dextran; Rh-ManBSA, rhodamine-labeled mannosylated BSA; MHC II, major histocompatibility complex-class II.

class II-positive cells that detached from the culture dish and accumulated in the culture medium between Day 1 and Day 4 were quantified and analyzed for their capacity to internalize fluorescent ligands. Data from a representative experiment are reported in Table 2 . The table shows the distribution of MHC class II-positive cells between the adherent and floating cell populations after 4 days of culture. Freshly dispersed cells were seeded in $6-\mathrm{cm}$ petri dishes at a density of $0.5 \times$ $10^{6}$ cells $/ \mathrm{cm}^{2}$ and cultured in the complete medium for 24 hours. The medium was then removed and replaced by the same, but serum-free, medium. Adherent cells at Day 1 and adherent and floating cells (cells present in the culture medium) at Day 4 were either collected for DNA assay or processed for MHC class II immunodetection. DNA measurements were converted into cell number, assuming that a cell contained 10 pg DNA. MHC class II-positive cells were counted in 10 microscope fields of adherent and floating cell preparations and expressed in percent of total cells determined by Hoechst labeling of nuclei. The absolute number and distribution of MHC class II-positive cells between the adherent and floating cell population at Day 4 were calculated from values of the total cell number obtained by DNA assay and values of the proportion of MHC class II-positive cells (in percent of total cell number). Results are the mean \pm SEM of triplicate. MHC class II-positive cells recovered in the culture media accounted for less than or about $15 \%$ of total MHC class II-positive cells in the culture dishes. Within the cell population collected from culture media by cytocentrifugation, up to $40 \%$ of cells were $\mathrm{MHC}$ class II-positive, but these cells exhibited low or no endocytic activity (Fig. 7).

Because the loss of cell adhesion to the culture substratum is a parameter indicating a transition from immature to mature DC, and because TNF $\alpha$ has been reported (Sallusto et al, 1995) to activate this process, we investigated whether this cytokine could alter (a) the distribution of DC between the adherent and floating cell populations and (b) the endocytic activity of DC. After 4 days of culture, cells were subjected to a TNF $\alpha$ treatment for 24 or 48 hours. Cells internalizing F-Dx and cells expressing $\mathrm{MHC}$ class II were quantified in both the adherent and the floating cell populations (Fig. 8). At the initiation of TNF $\alpha$ treatment (Hour 0 ), cells with a high F-Dx endocytic activity and expressing MHC class II molecules represented about $3 \%$ of the total adherent cell population. In the control condition (without $\operatorname{TNF} \alpha$ ), there was a time-dependent increase of the number of MHC class II-positive cells in the culture medium; they amounted to $17 \%$ and $35 \%$ of floating cells after 24 and 48 hours, respectively. Floating cells did not internalize F-Dx. Noteworthy, the fraction of DC that detached from the dish remained low because there was no significant change in the number of adherent DC. In the presence of TNF $\alpha$, there was a marked increase of both the absolute number and the proportion of $\mathrm{MHC}$ class II-positive cells in culture media. This demonstrates that TNF $\alpha$ selectively induced the detachment of DC and did not exert a general deleterious action on the adherent cell population. After 48 hours of TNF $\alpha$ treatment, MHC class II-positive cells accounted for the two-thirds of floating cells. As in control conditions, DC collected in the culture medium in response to TNF $\alpha$ had a very low or no endocytic activity. The TNF $\alpha$-induced DC detachment was associated with a significant reduction of the number of adherent DC (from 3\% to approximately $2 \%$ ), considering either $\mathrm{MHC}$ class II-positive cells or F-Dx-positive cells (Fig. 8).

Table 2. Distribution of MHC Class II-Positive Cells between the Adherent and Floating Cell Populations after 4 Days of Culture

\begin{tabular}{cccccc}
\hline & & & \multicolumn{3}{c}{ MHC class II-positive cells } \\
\cline { 4 - 5 } Day of & & Cell & Total cell number & & Absolute number \\
$\left(\times 10^{-3}\right)$ & population & $5300 \pm 150$ & $2.4 \pm 0.1$ & $127 \pm 5$ & Distribution \\
\hline \multirow{2}{*}{1} & Adherent cells & $5300 \pm 60$ & $2.4 \pm 0.3$ & $138 \pm 18$ & - \\
4 & Adherent cells & $5700 \pm 07 \%$ & $20 \pm 2$ & $13 \%$ \\
\hline
\end{tabular}




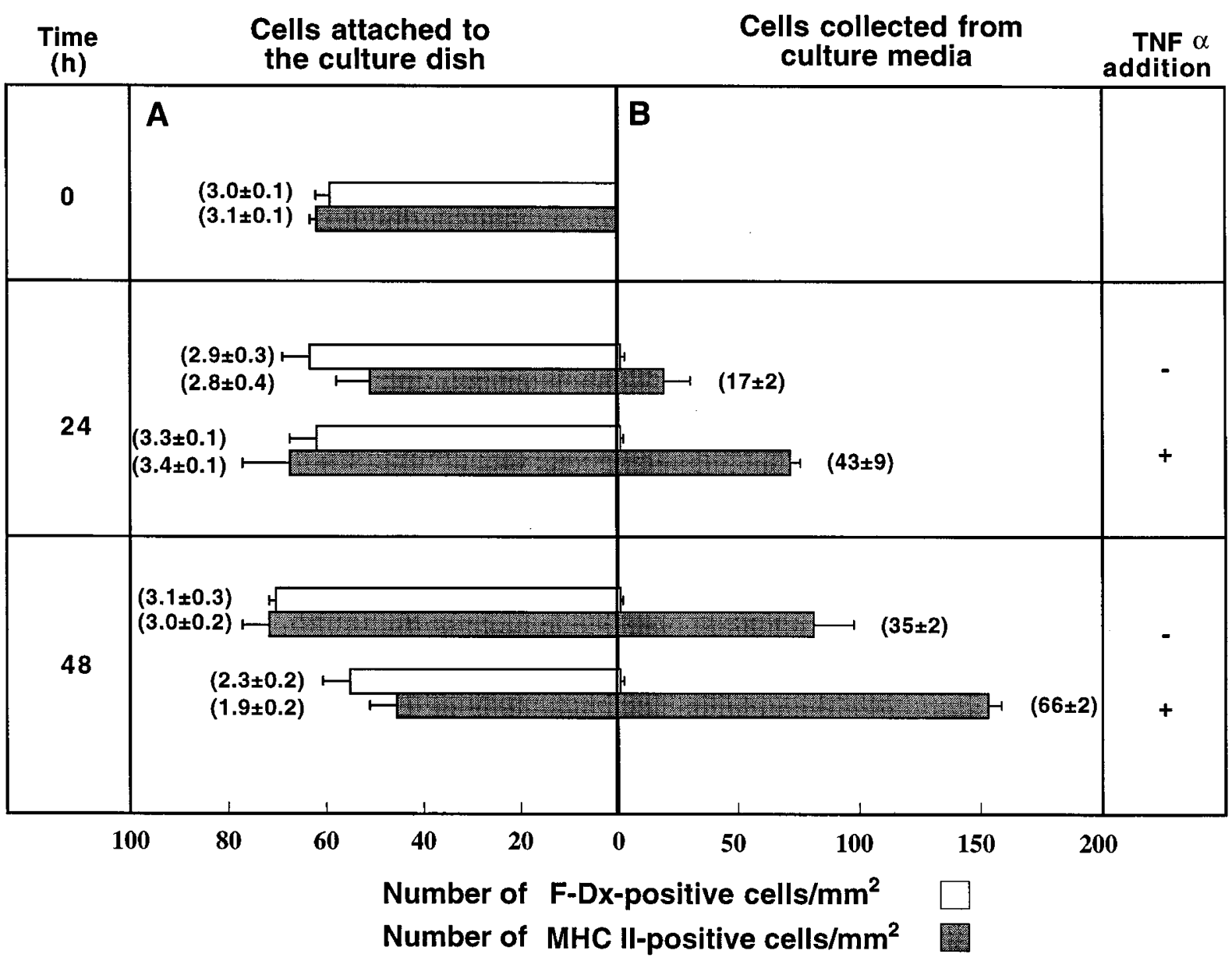

Figure 8.

Quantitative analyses of dendritic cells (DC) that detached from the culture dishes. Evidence for a TNF $\alpha$-induced change in the distribution of DC between the adherent and floating cell populations. After 4 days of culture, cells (thyrocytes and DC) were washed and further cultured in serum-free medium, with or without $10 \mathrm{ng} / \mathrm{ml}$ TNF $\alpha$, for 24 or 48 hours. After 21 and 45 hours of culture, F-Dx was added to the culture medium to assess the endocytic activity. Adherent cells and cells collected from the culture medium (and spread on glass slides) were immunolabeled with the anti-MHC class II antibody. F-Dx-labeled cells, MHC class II-positive cells, and Hoechst-labeled nuclei were counted on 10 microscope fields and values were used to calculate (a) the number of labeled cells per $\mathrm{mm}^{2}$ given by the columns of the histogram and (b) the proportion of labeled cells in percent of the total cell population (numbers in parentheses). Results are the mean \pm SEM of measurements on three dishes or glass slides.

\section{Discussion}

In recent years, DC have been the focus of intense research, and the knowledge of the molecular markers and functional characteristics of these cells has considerably increased (Banchereau and Steinman, 1998; Steinman, 1991). Hence, numerous immunological tools are now available to identify, select, and manipulate DC in humans and mice. In contrast, DC recognition in other species, such as the pig, is complicated by the lack of defined antibodies capable of reacting with DC markers. We have identified monoclonal antibodies directed against human antigens: $\mathrm{MHC}$ class II (HLA DR) molecules and the mannose receptor, that specifically reacted with the corresponding molecules in pigs. Using these antibodies and combinations of fluorescence labelings, we show that interstitial DC present in the thyroid gland can be isolated and maintained in coculture with thyroid epithelial cells in a ratio that is representative of the in vivo situation. Indeed, the proportion of DC estimated on tissue sections and quantified in the isolated cell population, as well as in short or more prolonged (up to 7 days) primary culture conditions, always ranged between $2 \%$ and $3 \%$.

Thyroid-derived DC in culture have been characterized on the basis of (a) morphological features (mainly a reniform nucleus and membrane processes that include veils, spikes, and fine dendrites), (b) phenotypic markers (MHC class II, mannose receptor, and $\mathrm{S} 100 \beta$ protein), and (c) their functional activity (endocytosis of labeled ligands). According to the present knowledge on DC (Banchereau and Steinman, 1998; Steinman, 1991), adherent, thyroid-derived DC in primary culture likely represent immature DC, ie, MHC class II cells equipped to capture antigens. The high endocytic activity of thyroid-derived DC probably depends on two distinct mechanisms of capture of exogenous molecules, as described for DC deriving from blood mononuclear cells (Sallusto et al, 1995). A first mechanism, related to the high expression of 
mannose receptor, would account for the internalization of carbohydrate-containing ligands (F-Dx, RhManSAB) (Sallusto et al, 1995; Sallusto and Lanzavecchia, 1994). Besides this receptor-mediated mode of uptake, DC can efficiently trap solutes by fluid-phase endocytosis (Sallusto and Lanzavecchia, 1994); we have found that DC were capable of internalizing BSA-gold particles. The mannose receptor, first described on macrophages (Shepherd et al, 1981) was more recently found on DC (Sallusto et al, 1995). It is a C-type lectin of $175 \mathrm{kDa}$ that contains multiple carbohydrate binding domains (Drickamer and Taylor, 1993; Stahl, 1992). This receptor is involved in the internalization of a variety of sugar-containing proteins (Pontow et al, 1992). On immature DC, the mannose receptor has been identified as the major receptor involved in the function of antigen presentation (Sallusto et al, 1995). Because of experimental limitations, we have been unable to perform the direct demonstration by double immunolabeling that thyroidderived MHC class II-positive cells are also mannose receptor-positive. Indeed, in the condition of fixation required for the mannose receptor immunodetection (ie, 70\% cold ethanol), the anti-MHC class II monoclonal antibody was ineffective. Nevertheless, data from the double labeling combinations reported in Table 1 indirectly indicate that the vast majority, if not all, of thyroid-derived DC (MHC class II- and S100 $\beta$ -positive cells) should express the mannose receptor. Western blot data show that cells expressing the mannose receptor are present in the pig, human, and rat thyroid glands. Immunolabelings on cryosections confirmed the presence of mannose receptor-positive cells in human thyroid tissue (unpublished data). The mannose receptor that was lost during the proteolytic treatment used for cell isolation was re-expressed in vitro. Indeed, mannose receptor-positive cells were detected within 2 days from the start of culture

It has been demonstrated that DC generated ex vivo from hematopoietic precursors and Langerhans cells readily shift in short-term culture from an immature to a mature state (Heufler et al, 1988; Steinman, 1991). This conversion or transition is accompanied by extensive functional transformations among which were the disappearance of the antigen-capturing activity and the migration into the culture medium. Thyroidderived DC (in coculture with thyrocytes) showed definite signs of maturation; a fraction of DC detached from the culture dishes, and these cells that remained strongly MHC class II-positive were devoid of endocytic activity. The ability of thyroid-derived DC to undergo maturation in vitro is further documented by the fact that the migration of DC from the adherent to the floating cell population and the concomitant change of endocytic status of detached DC are increased in response to TNF $\alpha$. Indeed, DC maturation is known to be controlled by various cytokines, including TNF $\alpha$ (Sallusto and Lanzavecchia, 1994; Steinman, 1991; Winzler et al, 1997). It is worth noticing that collection of cells from the culture media of thyrocytes/DC cocultures led to enriched populations of mature DC. After 48 hours of TNF $\alpha$ treatment, up to
$70 \%$ of floating cells were mature DC. Maturation of $\mathrm{DC}$ isolated from the thyroid gland differed, in many respects, from the maturation of DC generated from precursors. The phenotypic transformation of thyroidderived DC was slower, and only a fraction of cells underwent this change. These differences might be related to culture conditions. In this study, DC were cocultured with thyrocytes in the presence of serum for 24 hours to promote cell adhesion and then in serum-free medium. Serum was withdrawn, first of all, because it exerts adverse effects on in vitro morphological differentiation of thyrocytes, ie, follicle formation, and secondly, to enable us to test the action of exogenous factors on morphological and/or functional characteristics of DC in defined conditions. Previous studies on purified murine epidermal DC or Langerhans cells and human DC generated from $\mathrm{CD} 34^{+}$ progenitor cells have shown that the generation and/or survival, differentiation, and/or maturation of DC are dependent on cytokines, including GM-CSF, TNF $\alpha$, and TGF $\beta$ (Kocikova et al, 1998; Strobl et al, 1996, 1997). As thyroid-derived DC survived and exhibited differentiated functions in the absence of serum and without exogenous cytokines, it is reasonable to think that thyrocytes might produce factors endowed with regulatory actions on DC. Thyrocytes/DC interactions could be in some ways similar to the cytokine-mediated interactions between keratinocytes and Langerhans cells in coculture (Heufler et al, 1988).

In conclusion, our experimental approach might be used by others to isolate and analyze (a) DC from other organs in pigs and (b) DC from the thyroid gland from other species, especially from normal or pathological human thyroid tissues. Analyses of DC in pigs are of importance for studies aiming at the development of cell or organ systems for transfer or graft in humans. Applied to human thyroid tissue, this approach might contribute to new developments in thyroid autoimmunity studies. Further work is of course required to determine the immunological functions of thyroidderived DC maintained in culture.

\section{Materials and Methods}

\section{Materials}

Bovine thyrotropin (2 U/mg), fluorescein-labeled dextran (MM, $40 \mathrm{kDa})$, rhodamine-labeled mannosylated BSA, mouse immunoglobulins, the mouse monoclonal antibovine $\mathrm{S} 100 \beta$ antibody, sheep antimouse IgG, affinity-purified $F\left(a b^{\prime}\right)_{2}$ fragments conjugated to Texas red or FITC were obtained from Sigma Chemical Company (St. Louis, Missouri). Tissue Tech OCT compound was purchased from Miles Inc. (Elkart, Indiana). Rabbit antipig thyroglobulin antibodies generated in our laboratory had been used in previous studies (Selmi and Rousset, 1988). The mouse monoclonal antibody (15-2-2) directed against the human mannose receptor was a gift from Dr. D. C. Rijken, Gaubius Laboratory, Leiden, The Netherlands. The anti-MHC class II antibody was a mouse monoclonal antihuman 
human leukocyte antigen (HLA) class II (DR) antibody obtained from Novocastra Laboratory (Newcastle, United Kingdom). Donkey antirabbit IgG antibodies coupled to Texas red were obtained from Amersham (Buckinghamshire, United Kingdom). Goat anti-mouse $\operatorname{lgG}, F\left(a b^{\prime}\right)_{2}$ specific antibodies conjugated to either Texas red or FITC and $F(a b)$ fragments of goat IgG anti-mouse Ig were obtained from Jackson Immunoresearch (West Grove, Pennsylvania). The Hoechst 33342 reagent was purchased from Molecular Probes (Leiden, The Netherlands).

\section{Preparation of the Thyroid Cell Suspension and Culture Conditions}

Thyroid glands from adult pigs were obtained from the local slaughterhouse and processed within 1 hour after animal death. Glands were trimmed free from fat and connective tissue and chopped into small pieces of 50 to $100 \mathrm{~mm}^{3}$. Minced tissue $(80-100 \mathrm{~g})$ was washed three times in cold $\left(4^{\circ} \mathrm{C}\right)$ Earle's solution and placed in a flask containing $250 \mathrm{ml}$ of trypsin solution (trypsin $0.25 \%$ in Earle's balanced salt solution at $\mathrm{pH}$ 7.5). Trypsinization was carried out at $37^{\circ} \mathrm{C}$ for five successive periods of 30 minutes each under constant stirring. After each period the cell suspension was filtered through a steel sieve into 250-ml centrifuge bottles containing $10 \mathrm{ml}$ of calf serum and the filtrate was centrifuged at $4^{\circ} \mathrm{C}$ for 10 minutes at 80 to 100 $\times g$. Cell pellets were then resuspended and washed once in calf serum, then twice in Earle's solution, and filtered through four layers of gauze, with 80 to $100 \mathrm{~g}$ of thyroid tissue usually yielding about $1 \mathrm{ml}$ of packed cells corresponding to $10^{9}$ cells. The cell suspension resulting from the proteolytic enzyme treatment was composed of isolated cells and clusters of few cells. After extensive washing of the cell suspension in Ham's F-12 medium, cells were seeded at a density of $0.5 \times 10^{6}$ cells $/ \mathrm{cm}^{2}$ in $60-\mathrm{mm}$ tissue culture-treated petri dishes in Ham's F-12 medium, supplemented with $5 \%$ calf serum, penicillin $(100 \mathrm{U} / \mathrm{ml})$, streptomycin $(0.1 \mathrm{mg} / \mathrm{ml})$, fungizone $(0.25 \mu \mathrm{g} / \mathrm{ml})$, and thyrotropin (1 $\mathrm{mU} / \mathrm{ml})$.

After one day of culture, the medium was replaced by the same but serum-free medium. Except where otherwise stated, there was no other medium change. Cells were cultured at $37^{\circ} \mathrm{C}$ in a $5 \% \mathrm{CO}_{2}$-air atmosphere for up to 7 days. Under these conditions, thyrocytes reorganize into three-dimensional structures named in vitro reconstituted thyroid follicles as previously described (Gire et al, 1996; Munari-Silem et al, 1990; Pellerin et al, 1999).

\section{Preparation of Cryosections from Pig Thyroid Tissue}

Small pieces of pig thyroid tissue were cut immediately after collection of the organ, embedded in Tissue-Teck OCT compound, and frozen in liquid nitrogen. Tissue sections (5- $\mu \mathrm{m}$ thick), prepared at $-20^{\circ} \mathrm{C}$ using a Reichert-Jung cryostat (Vienna, Austria), were mounted on silaned slides and stored at $-20^{\circ} \mathrm{C}$ until use.

\section{Immunolabeling Procedures}

Tissue sections and cultured cells were fixed either in $70 \%$ cold ethanol for 30 minutes at $4^{\circ} \mathrm{C}$ for mannose receptor immunodetection or in $4 \%$ paraformaldehyde in PBS for 30 minutes at room temperature for all other immunofluorescence labelings. Paraformaldehyde-fixed cells or tissue sections were permeabilized in $0.05 \%$ Tween 20 or $0.5 \%(\mathrm{p} / \mathrm{v})$ saponin for $\mathrm{MHC}$ class II detection in PBS for 30 minutes at room temperature. Cultured cells and tissue sections were preincubated for 1 hour at room temperature in PBS containing $10 \mathrm{mg} / \mathrm{ml}$ BSA (PBS-BSA) or $3 \%$ sheep nonimmune serum and incubated for 1 hour at $37^{\circ} \mathrm{C}$ with the appropriate primary antibody. The rabbit antipig thyroglobulin antiserum was used at a final dilution of $1 / 300$. The monoclonal antibody directed against the mannose receptor was used at a concentration of $0.1 \mu \mathrm{g} / \mathrm{ml}$. Monoclonal antibodies against $\mathrm{MHC}$ class II and $\mathrm{S} 100 \beta$ were used at dilutions of $1 / 200$ and $1 / 500$, respectively. Immune complexes were detected with either donkey antirabbit lgG antibodies coupled to Texas red or with goat antimouse $\operatorname{lgG}, F\left(a b^{\prime}\right)_{2}$ specific antibodies conjugated to either Texas red or FITC. In the case of tissue cryosection labelings, immune complexes were detected with sheep antimouse lgG, affinity-purified $F\left(a b^{\prime}\right)_{2}$ fragments conjugated to either Texas red or FITC. All conjugates were used as indicated by the manufacturers. Nuclei staining was obtained by incubating cells with $2 \mu \mathrm{g} / \mathrm{ml}$ Hoechst 33342 for 15 minutes at room temperature.

The double immunodetection of $\mathrm{MHC}$ class II and $\mathrm{S} 100 \beta$ protein was performed using a previously reported method (Negoescu et al, 1994) that allowed us to use two primary antibodies from the same species, ie, two mouse monoclonal antibodies. Briefly, in a first step, cells were incubated with the anti-MHC class II monoclonal antibody (first primary antibody) and then with Texas red-labeled goat antimouse lg antibodies (first secondary antibody) as described above. In the second step, cells were incubated with mouse immunoglobulins $(1 \mathrm{mg} / \mathrm{ml})$ for 1 hour at room temperature to achieve saturation of residual antigen binding sites on the first secondary antibody and then overnight at $4^{\circ} \mathrm{C}$ with $\mathrm{F}(\mathrm{ab})$ fragments of goat $\lg \mathrm{G}$ antimouse $\lg (0$, $1 \mathrm{mg} / \mathrm{ml}$ ) to saturate antibody binding sites on the first primary antibody. The third step consisted in the immunolabeling of $S 100 \beta$ protein using the anti$\mathrm{S} 100 \beta$ monoclonal antibody (second primary antibody) and fluorescein-labeled goat antimouse lg antibodies (second secondary antibody). Controls included the replacement of either the first primary antibody, the second primary antibody, or the first secondary antibody by PBS-BSA. The specificity of the two-step labeling was demonstrated by a distinct cellular localization of the red (MHC class II) and green (S100 $\beta$ ) fluorescence.

\section{Endocytosis of Fluorescent Ligands}

Cells were incubated with fluorescein-labeled dextran ( $1 \mathrm{mg} / \mathrm{ml}$ ) and/or Rh-ManBSA ( $1 \mathrm{mg} / \mathrm{ml})$ for 3 hours at $37^{\circ} \mathrm{C}$. At the end of the incubation period, culture 
media were collected and adherent cells were washed three times with cold Earle's medium, $\mathrm{pH} 7$, and fixed either in $70 \%$ cold ethanol for 30 minutes at $4^{\circ} \mathrm{C}$ or in $4 \%$ paraformaldehyde in PBS for 30 minutes at room temperature for subsequent quantitative fluorescence analyses and immunolabelings. Cells present in culture media (floating cells) were pelleted by centrifugation at $400 \times g$ for 10 minutes, resuspended in 100 to $300 \mu \mathrm{l}$ undiluted calf serum and spread on glass slides by cytocentrifugation at $50 \times g$ for 10 minutes using a cytospin centrifuge from Shandon Elliott (Camberley, United Kingdom). Cells on glass slides were submitted to the same fixation and labeling protocols as those used for adherent cells.

\section{Endocytosis of Protein-Gold Complexes and Processing for Electron Microscopy}

BSA-gold and thyroglobulin-gold complexes were prepared from 9-nm and 15-nm gold particles, respectively, as previously described (Kostrouch et al, 1993). Cells were incubated with a 1 to 1 mixture of BSA-gold and thyroglobulin-gold complexes $\left(10^{13}\right.$ to $10^{14}$ particles/ml) in Ham's F-12 medium containing BSA $(1 \mathrm{mg} / \mathrm{ml})$ for 60 minutes at $37^{\circ} \mathrm{C}$ as previously described (Gire et al, 1996). Cells were then washed and fixed with $2 \%$ glutaraldehyde in $0.1 \mathrm{M}$ cacodylate buffer, $\mathrm{pH} 7.4$, for 40 minutes and postfixed in $1 \%$ osmium tetroxide in $0.15 \mathrm{M}$ cacodylate buffer for 20 minutes. Cells were then dehydrated and embedded in Epon resin. Ultrathin sections were made using a diamond knife and contrasted with uranyl acetate and lead citrate. Observations were made using a JEOL 1200 EX electron microscope (Centre Commun d'Imagerie Laennec, Faculté de Médecine Lyon-RTH Laennec, Lyon, France).

\section{Videomicroscope Fluorescence Image Processing and Quantitative Analyses}

Fluorescence images, obtained with a Zeiss Axiophot fluorescence microscope equipped with the following filter combinations (excitation-emission): G 365 LP 420 for Hoechst, BP 615 LP 590 for Texas red and rhodamine, and BP 450-490 LP 520 for FITC, were monitored using a cooled CCD camera (Lhesa Electronique, Cergy Pontoise, France). Images were transferred to a 80486 IBM compatible personal computer equipped with a Cyclope imaging card (Digital Vision, Chatillon, France). Software was designed for imagenoise reduction and pseudocolor restitution. In the case of double- or triple-labeling with fluorescein, Texas red or Rhodamine, and Hoechst, fluorescence images corresponding to each fluorophore were recorded sequentially. Fluorescence images, as well as phase-contrast images, were printed on paper using a UP-5000P video printer from Sony (Tokyo, Japan). It was verified that there was no mechanically induced shift during the procedure of sequential image collection. Quantitative analyses of cells labeled after endocytosis of a fluorescent ligand or immunolabeled with a given antibody were performed on 10 or 20 micro- scope fields taken at random in each culture dish using the $\times 63$ objective. Numbering of labeled cells and Hoechst-labeled nuclei (giving the total number of cells) in a given field was carried out by manual counting on a monitor screen. Values were used to calculate the average number of labeled cells per $\mathrm{mm}^{2}$ and/or the proportion of labeled cells (in percent of total cells) in the culture dish. Measurements were made on duplicate or triplicate dishes.

\section{Other Methods}

Protein was assayed by the Lowry method using a pretreatment by $0.1 \%$ deoxycholate to solubilize membrane proteins and BSA as standard. DNA assay was performed using the fluorometric method of Labarca and Paigen (1980) with the Hoechst reagent and calf thymus DNA as standard. Western blot was carried out as previously described (Riou et al, 1999).

\section{Acknowledgements}

We thank Dr. D.C. Rijken (Gaubius Laboratory, TNO Prevention and Health, Leiden, The Netherlands) for providing us with the anti-mannose receptor monoclonal antibody and Mrs. A. Callé and Mrs. I. Berger for their technical assistance in the preparation of thyroid cryosections.

\section{References}

Banchereau J and Steinman RM (1998). Dendritic cells and the control of immunity. Nature 392:245-252.

Caux C, Vanbervliet B, Massacrier C, Dezutter-Dambuyant C, de Saint-Vis B, Jacquet C, Yoneda K, Imamura S, Schmitt D, and Banchereau J (1996). CD34+ hematopoietic progenitors from human cord blood differentiate along two independent dendritic cell pathways in response to GM-CSF+TNF $\alpha$. J Exp Med 184:695-706.

Drickamer K and Taylor ME (1993). Biology of animal lectins. Annu Rev Cell Biol 9:237-264.

Gire V, Kostrouch Z, Bernier-Valentin F, Rabilloud R, MunariSilem Y, and Rousset B (1996). Endocytosis of albumin and thyroglobulin at the basolateral membrane of thyrocytes organized in follicles. Endocrinology 137:522-532.

Hart DNJ and Fabre JW (1981). Demonstration and characterization of la-positive dendritic cells in the interstitial connective tissues of rat heart and other tissues, but not brain. $J$ Exp Med 153:347-361.

Hart DNJ, Newton MR, Reece-Smith H, Fabre JW, and Morris PJ (1983). Major histocompatibility complex antigens in the rat pancreas, isolated pancreatic islets, thyroid, and adrenal. Transplantation 36:431-435.

Heufler C, Koch F, and Schuler G (1988). Granulocyte/ macrophage colony-stimulating factor and interleukin 1 mediate the maturation of murine epidermal Langerhans cells into potent immunostimulatory dendritic cells. J Exp Med 167:700-705.

Kabel PJ, Voorbij HAM, de Haan M, Van der Gaag RD, and Drexhage HA (1988). Intrathyroidal dendritic cells. J Clin Endocrinol Metab 66:199-207. 
Knight SC, Farrant J, Chan J, Bryant A, Bedford PA, and Bateman C (1988). Induction of autoimmunity with dendritic cells: Studies of thyroiditis in mice. Clin Immunol Immunopathol 48:277-289.

Kocikova A, Kolesaric A, Koszik F, Stingl G, and ElbeBurger A (1998). Murine Langerhans cells cultured under serum-free conditions mature into potent stimulators of primary immune responses in vitro and in vivo. $J$ Immunol 161:4033-4041.

Kostrouch Z, Bernier-Valentin F, Munari-Silem Y, Rajas F, Rabilloud R, and Rousset B (1993). Thyroglobulin molecules internalized by thyrocytes are sorted in early endosomes and partially recycled back to the follicular lumen. Endocrinology 132:2645-2653.

Labarca C and Paigen K (1980). A simple, rapid, and sensitive DNA assay procedure. Anal Biochem 102:344-352.

Lennartz MR, Cole FS, and StahI PD (1989). Biosynthesis and processing of the mannose receptor in the human macrophages. J Biol Chem 264:2385-2390.

Many MC, Maniratunga S, Varis L, Dardenne M, Drexhage HA, and Denef JF (1995). Two-step development of Hashimoto-like thyroiditis in genetically autoimmune prone non-obese diabetic mice: Effects of iodine-induced cell necrosis. J Endocrinol 147:311-320.

Martinez-Pomares L, Mahoney JA, Kaposzta R, Linehan SA, Stahl PD, and Gordon S (1998). A functional soluble form of the murine mannose receptor is produced by macrophages in vitro and is present in mouse serum. $\mathrm{J}$ Biol Chem 273: 23376-23380.

Masten BJ and Lipscomb MF (1999). Comparison of lung dendritic cells and $B$ cells in stimulating naive antigenspecific T cells. J Immunol 162:1310-1317.

Mölne J, Jansson S, Ericson LE, and Nilsson M (1994). Adherence of RFD-1 positive dendritic cells to the basal surface of thyroid follicular cells in Graves'disease. Autoimmunity 17:59-71.

Munari-Silem Y, Mesnil M, Selmi S, Bernier-Valentin F, Rabilloud R, and Rousset B (1990). Cell-cell interactions in the process of differentiation of thyroid epithelial cells into follicles: A study by microinjection and fluorescence microscopy on in vitro reconstituted thyroid follicles. J Cell Physiol 145:414-427.

Negoescu A, Labat-Moleur F, Lorimier P, Lamarcq L, Guillermet C, Chambaz E, and Brambilla E (1994). F(ab) secondary antibodies: A general method for double immunolabeling with primary antisera from the same species. Efficiency control by chemiluminescence. J Histochem Cytochem 42: 433-437.

Pellerin S, Croizet K, Rabilloud R, Feige JJ, and Rousset B (1999). Regulation of the three-dimensional organization of thyroid epithelial cells into follicle structures by the matricellular protein, thrombospondin-1. Endocrinology 140:1094-1103.

Pontow SE, Kery V, and Stahl PD (1992). Mannose receptor. Int Rev Cytol 137B:221-244.

Riou C, Tonoli H, Bernier-Valentin F, Rabilloud R, Fonlupt $\mathrm{P}$, and Rousset B (1999). Susceptibility of differentiated thyrocytes in primary culture to undergo apoptosis after exposure to hydrogen peroxyde: Relation with the level of expression of apoptosis regulatory proteins, $\mathrm{Bcl}-2$ and Bax. Endocrinology 140:1990-1997.
Sallusto F, Cella M, Danieli C, and Lanzavecchia A (1995). Dendritic cells use macropinocytosis and the mannose receptor to concentrate macromolecules in the major histocompatibility complex class II compartments: Downregulation by cytokines and bacterial products. J Exp Med 182: 389-400.

Sallusto F and Lanzavecchia A (1994). Efficient presentation of soluble antigen by cultured human dendritic cells is maintained by granulocyte/macrophage colony-stimulating factor plus interleukin 4 and downregulated by tumor necrosis factor $\alpha$. J Exp Med 179:1109-1118.

Schuler G and Steinman RM (1985). Murine epidermal Langerhans cells mature into potent immunostimulatory dendritic cells in vitro. J Exp Med 161:526-546.

Selmi S and Rousset B (1988). Identification of two subpopulations of thyroid lysosomes. Relation to the thyroglobulin proteolytic pathway. Biochem J 253:523-532.

Shepherd VL, Lee YC, Schlesinger PH, and Stahl PD (1981). L-fucose-terminated glycoconjugates are recognized by pinocytosis receptors on macrophages. Proc Natl Acad Sci U S A 78:1019-1022.

Simons PJ, Delemarre FGA, and Drexhage HA (1998). Antigen-presenting dendritic cells as regulators of the growth of thyrocytes: A role of interleukin- $1 \beta$ and interleukin-6. Endocrinology 139:3148-3156.

Stahl PD (1992). The mannose receptor and other macrophages lectins. Curr Opin Immunol 4:49-52.

Stahl PD, Schlesinger PH, Sigardson E, Rodman JS, and Lee YC (1980). Receptor-mediated pinocytosis of mannose glycoconjugates by macrophages: Characterization and evidence for receptor recycling. Cell 19:207-215.

Steinman RM (1991). The dendritic cell system and its role in immunogenicity. Annu Rev Immunol 9:271-296.

Strobl H, Bello-Fernandez C, Riedl E, Pickl WF, Majdic O, Lyman SD, and Knapp W (1997). flt3 ligand in cooperation with transforming growth factor- $\beta 1$ potentiates in vitro development of Langerhans-type dendritic cells and allows singlecell dendritic cell cluster formation under serum-free conditions. Blood 90:1425-1434.

Strobl H, Riedl E, Scheinecker C, Bello-Fernandez C, Pickl WF, Rappersberger K, Majdic O, and Knapp W (1996). TGF-beta 1 promotes in vitro development of dendritic cells from CD34+ hemopoietic progenitors. J Immunol 157:14991507.

Voorby HA, Kabel PJ, De Hann M, Jeucken PH, Van der Gaag RD, De Baets MH, and Drexhage HA (1990). Dendritic cells and class II MHC expression on thyrocytes during autoimmune thyroid disease of BB rat. Clin Immunol Immunopathol 55:9-22.

Winzler C, Rovere P, Rescigno M, Granucci F, Penna G, Adorini L, Zimmermann VS, Davoust J, and RicciardiCastognoli P (1997). Maturation stages of mouse dendritic cells in growth factor-dependent long-term cultures. J Exp Med 185:317-328. 